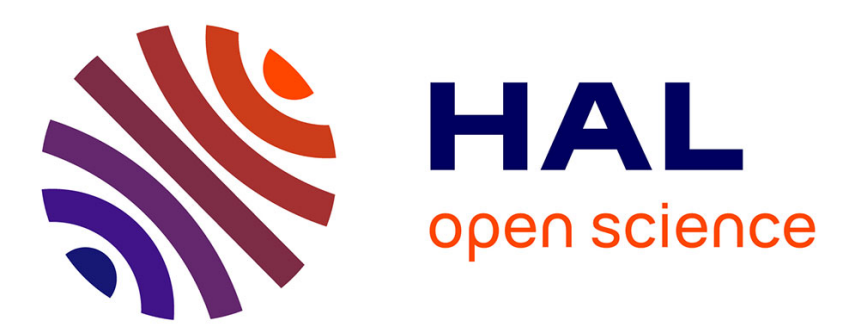

\title{
Analytical calculation of the flux density distribution in a superconducting reluctance machine with HTS bulks rotor
}

Gaël Malé, Thierry Lubin, Smail Mezani, Jean Lévêque

\section{- To cite this version:}

Gaël Malé, Thierry Lubin, Smail Mezani, Jean Lévêque. Analytical calculation of the flux density distribution in a superconducting reluctance machine with HTS bulks rotor. Mathematics and Computers in Simulation, 2013, 13 p. 10.1016/j.matcom.2013.01.003 . hal-00833958

\section{HAL Id: hal-00833958 \\ https://hal.science/hal-00833958}

Submitted on 13 Jun 2013

HAL is a multi-disciplinary open access archive for the deposit and dissemination of scientific research documents, whether they are published or not. The documents may come from teaching and research institutions in France or abroad, or from public or private research centers.
L'archive ouverte pluridisciplinaire HAL, est destinée au dépôt et à la diffusion de documents scientifiques de niveau recherche, publiés ou non, émanant des établissements d'enseignement et de recherche français ou étrangers, des laboratoires publics ou privés. 


\title{
Analytical calculation of the flux density distribution in a superconducting reluctance machine with HTS bulks rotor
}

\author{
Gael Malé, Thierry Lubin*, Smail Mezani and Jean Lévêque \\ Université de Lorraine, Groupe de Recherche en Electrotechnique et Electronique de Nancy, GREEN \\ F-54500 Vandoeuvre-lès-Nancy, France
}

\begin{abstract}
This paper deals with the analytical computation of the magnetic field distribution in a wholly superconducting reluctance motor. The rotor is made with high temperature superconductor bulks which nearly present a diamagnetic behavior under zero-field cooling. The stator consists of superconducting armature windings fed by AC currents of high amplitude. The superconducting stator winding can generate a high rotating magnetic field without the need of ferromagnetic material in the rotor. The electromagnetic torque is obtained by the interaction between the rotating magnetic field created by the superconducting stator windings and the variable reluctance due to the superconducting bulks. The proposed analytical method is based on the resolution of Laplace's equation (by the separation of variables method) for each sub-domain, i.e. rotor shaft, holes between superconducting bulks and air-gap. The global solution is obtained using boundary and continuity conditions. Magnetic field distribution and electromagnetic torque obtained by the analytical method are compared with those obtained from finite element analyses.
\end{abstract}

\section{Introduction}

High temperature superconducting (HTS) materials can be used in various types of electrical devices like motors and generators which represent an important part of the superconducting applications [15]. Among them, Superconducting Synchronous Reluctance Motors (ScSynRM) are studied and tested by different authors as, Chu and Torii [5], Kovalev et al. [9], Malé et al. [12], and Oswald et al. [14].

Conventional synchronous reluctance motors (SynRM) with ferromagnetic materials present a simple structure and rugged characteristics [2]. The rotor of such motors consists of magnetic and nonmagnetic materials. The electromagnetic torque depends on the difference between the $d$ - and $q$-axis inductances $\left(L_{d}-L_{q}\right)$. Recently, some authors have proposed to replace the nonmagnetic materials by HTS bulks. HTS bulks present a diamagnetic behavior when they are zero-field cooling [14]. This property is used to decrease the $q$-axis inductance of reluctance motors in order to improve the performances of such motors.

In this paper, we propose an analytical modeling of a wholly HTS ScSynRM without ferromagnetic materials in the rotor. Compared to [9] and [14], the proposed motor uses superconducting materials for both the stator and the rotor. Fig. 1 shows the structure of the studied motor. To simplify the analytical model, the HTS stator windings will be approximated by an equivalent current sheet placed on the stator bore as shown in Fig. 1. In addition, we will consider the superconductor materials as ideals (no AC losses in the HTS coils and perfect diamagnetic behavior of the HTS bulks).

The performances of the studied superconducting reluctance motor would be analyzed by an analytical model developed in this paper. Analytical methods are, in general, less computational time consuming than numerical ones (like finite-element method) and can provide closed-form solutions giving physical insight for designers. They can be useful tools for the first step of design optimization. Here, the two-dimensional Laplace equation is solved in each sub-domain (rotor shaft, holes between HTS bulks, and airgap). The solution in each region is obtained using boundaries and interfaces conditions.

The paper is organized as follows. The problem description and the assumptions of the model are presented in section 2. Section 3 describes the analytical method for magnetic field calculation in the different subdomains. The analytical results are then verified with finite-element method in section 4 .

\footnotetext{
* Corresponding author. Tel.: +33 (0)3 836841 27; fax: +33 (0)3 83684133

E-mail address: thierry.lubin@univ-lorraine.fr
} 


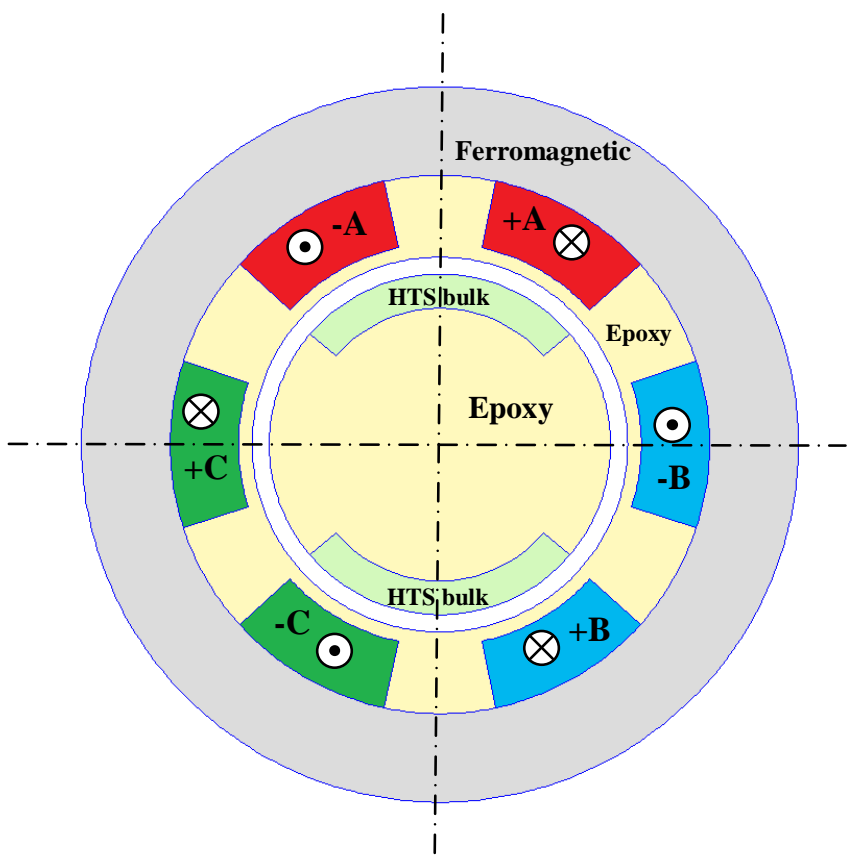

(a)

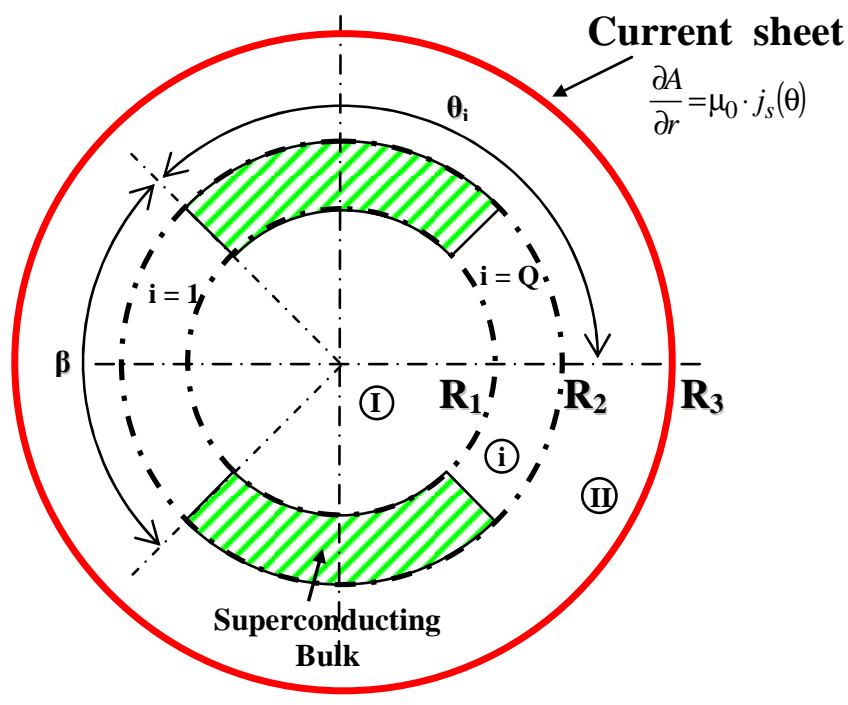

(b)

Fig. 1. Superconducting reluctance machine: (a) Stucture, (b) Simplified model $\left(Q=2, p=1, \theta_{0}=0\right)$.

\section{Problem description and assumptions}

The structure of the studied ScSynRM is shown in Fig. 1a. It consists of a three-phase concentrated HTS stator winding and $Q$ HTS bulks placed in the rotor. In the HTS stator, the epoxy teeth insure the mechanical stiffness of the structure. The stator yoke acts as a magnetic shield, so its height is chosen to avoid magnetic saturation. In order to simplify the analysis, the HTS stator winding is approximated by an equivalent $p$ polepairs current sheet placed on the stator bore as shown in Fig. 1b. This current sheet should create the same fundamental component of the airgap flux density as the real HTS winding. The rotor contains a ring of $Q$ holes sub-domains (region $i, i=1$ to $Q$ ) between the $Q$ HTS bulks.

The magnetic field generated by the stator interacts with the HTS bulks rotor. The electromagnetic torque is obtained by the magnetic flux lines deviation due to the flux shielding capability of the HTS bulks rotor.

Analytical approaches for the magnetic field computation in classical ferromagnetic machines have been studied recently by many authors as Bellara et al. [1], Dubas and Espanet [6], Liu and Li [10], Chebak et al. [4], Tiegna et al. [16], Zhu et al. [17] for permanent magnets machines and Lubin et al. [11] for reluctance motor. A general formulation has been proposed by Gysen et al. [8]. However, the proposed analytical models are related 
to iron-cored electrical machine (with perfect ferromagnetic boundary conditions to take into account the slotting effect) and cannot be used for the analysis of the proposed machine with HTS bulks. Therefore, it is necessary to develop a new analytical model for the analysis of this kind of motor. The mathematical approach developed in the paper is similar to the one proposed in [8]. It is based on boundary value problems with Fourier analysis. However, compared to previous works, we have to consider perfect diamagnetic boundary conditions (Dirichlet boundary conditions) in the boundary value problems to take into account the effect of the HTS bulks on the magnetic field distribution.

The geometrical parameters of the studied HTS motor are:

- for the rotor, inner and outer radii of the bulks $R_{1}$ and $R_{2}$ respectively,

- for the equivalent current sheet, the radius $R_{3}$ of the stator bore.

As indicated previously, the three-phase stator winding is represented by a current sheet distributed over the stator bore. The studied HTS reluctance machine being a synchronous one, the static torque is obtained by moving the rotor while keeping the magnetic field created by the stator currents at a given position. In this case, the current sheet is written as:

$$
j_{s}(\theta)=J_{0} \cos (p \theta)
$$

where $J_{0}$ is the peak value of the current sheet in $(\mathrm{A} / \mathrm{m})$.

The hole opening angle is $\beta$. The angular position of the $i$-th hole is defined as:

$$
\theta_{i}=-\frac{\beta}{2}+\frac{2 i \pi}{Q}+\theta_{0} \text { with } 1 \leq i \leq Q
$$

where $\theta_{0}$ is the initial angular position of the rotor

In order to derive the analytical model, some assumptions are made:

- The machine is supposed infinitely long in the $\mathrm{z}$ direction i.e. end effects are neglected.

- Infinite permeability of the stator back iron,

- HTS bulks have radial sides.

- Perfect diamagnetic behavior of HTS bulks.

Fig. 2a shows the flux lines distribution around a superconducting bulk when it is cooled down below its critical temperature $T_{c}$ before a magnetic field is applied. The superconducting material is modeled by a powerlaw which links the electric field $E$ to the current density $J ; E(J)=E_{c}\left(J / J_{c}\right)^{n}$, where $J_{c}$ is the critical current density, $E_{c}$ is the critical electric field $\left(10^{-4} \mathrm{~V} / \mathrm{m}\right)$ and $n$ is the power law index [3]. As it can be observed, the flux is repelled from the HTS bulk except on the edges. This is due to the presence of surface currents in the HTS bulk which prevent the penetration of the external magnetic field (Faraday's law). The penetration of the flux inside the bulk depends on the superconductor characteristics, on the temperature cooling, and on the magnitude of the applied magnetic field. The characteristics of HTS windings (DI-BSCCO) and HTS bulks (YBaCuO) can be found in [18] and [19]. Fig. 2b shows the flux lines while assuming a perfect diamagnetic behavior of the HTS bulk. Compared to Fig. 2a, the flux is totaly rejected from the HTS bulk and the flux lines are tangential to the surface of the bulk. In terms of boundary conditions, we can set $A=0$ on the surface of the bulk (the normal component of the flux density on the boundaries of the HTS bulk is null i.e. $\boldsymbol{B} . \boldsymbol{n}=0)$. Calculations in this paper will neglect field penetration into the superconductor. So, The boundary condition $A=0$ on all superconducting surfaces will be used in the next sub-section to derive the proposed analytical model.

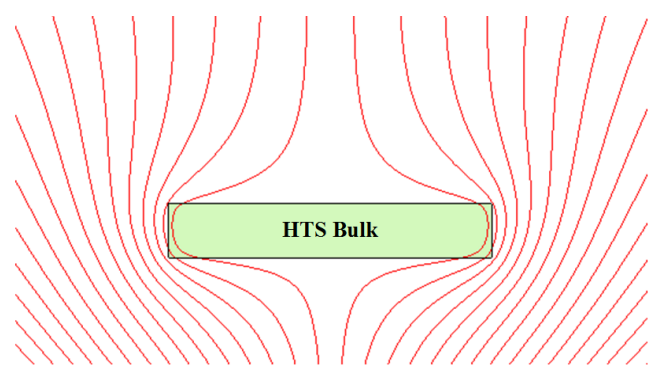

(a)

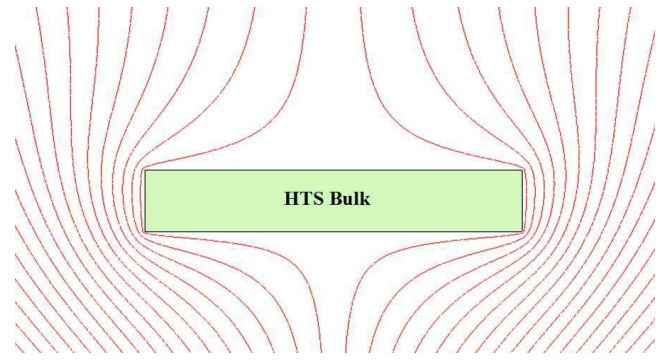

(b)

Fig. 2. Flux lines when the external field is applied after the cooling: (a) "real" HTS Bulk with $\mathrm{E}_{\mathrm{c}}=10^{-4} \mathrm{~V} / \mathrm{m}, \mathrm{J}_{\mathrm{c}}=100 \mathrm{~A} / \mathrm{mm}{ }^{2} \mathrm{and} \mathrm{n}=30$, (b) "perfect" HTS Bulk. 


\section{Problem formulation and solution}

A magnetic vector potential formulation is used in 2D polar coordinates to describe the problem. According to the adopted assumptions, the magnetic vector potential $\boldsymbol{A}$ presents only one component along the $z$-direction and only depends on the $r$ and $\theta$ coordinates. The notations used in the paper are:

$$
\begin{aligned}
& \boldsymbol{A}_{\boldsymbol{I}}=A_{I}(r, \theta) \boldsymbol{e}_{z} \text { for the shaft sub-domain } \\
& \boldsymbol{A}_{\boldsymbol{i}}=A_{i}(r, \theta) \boldsymbol{e}_{z} \text { for the } i \text {-th hole sub-domain } \\
& \boldsymbol{A}_{I I}=A_{I I}(r, \theta) \boldsymbol{e}_{z} \text { for the air-gap sub-domain }
\end{aligned}
$$

For simplicity and more clarity of the general solutions in the different sub-domains, the following notations are adopted throughout the paper:

$$
\begin{aligned}
& \mathrm{P}_{u}\left(R_{v}, R_{w}\right)=\left(\frac{R_{v}}{R_{w}}\right)^{u}+\left(\frac{R_{v}}{R_{w}}\right)^{-u} \\
& E_{u}\left(R_{v}, R_{w}\right)=\left(\frac{R_{v}}{R_{w}}\right)^{u}-\left(\frac{R_{v}}{R_{w}}\right)^{-u}
\end{aligned}
$$

\subsection{General solution of Laplace's equation in the $i$-th hole sub-domain (region i)}

Fig. 3 shows the $i$-th hole sub-domain and its associated boundary conditions. The Laplace's equation must be solved in a domain of inner radius $R_{l}$ and outer radius $R_{2}$ delimited by the angles $\theta_{i}$ and $\theta_{i}+\beta$.

$$
\frac{\partial^{2} A_{i}}{\partial r^{2}}+\frac{1}{r} \frac{\partial A_{i}}{\partial r}+\frac{1}{r^{2}} \frac{\partial^{2} A_{i}}{\partial \theta^{2}}=0 \quad \text { for } \quad\left\{\begin{array}{l}
R_{1} \leq r \leq R_{2} \\
\theta_{i} \leq \theta \leq \theta_{i}+\beta
\end{array}\right.
$$

Considering a diamagnetic behavior for the HTS bulks, the normal component of the magnetic field at the sides of the HTS bulk is null. The boundary conditions for the $i$-th hole domain are:

$$
\left.A_{i}\right|_{\theta=\theta_{i}}=0 \quad \text { and }\left.\quad A_{i}\right|_{\theta=\theta_{i}+\beta}=0
$$

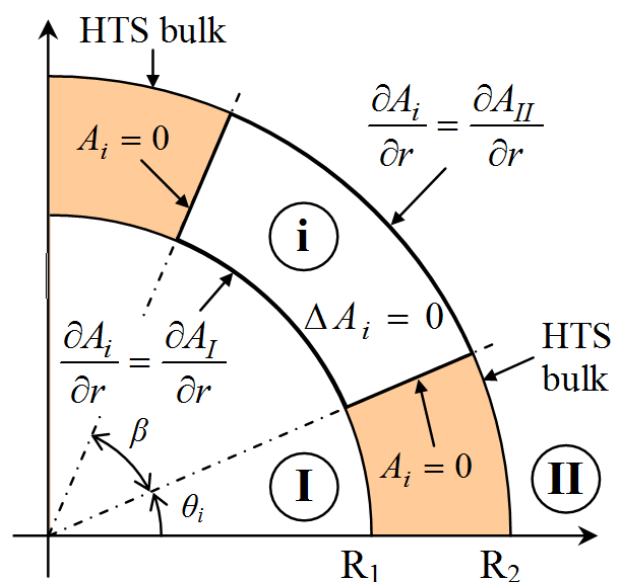

Fig. 3. $i$-th hole sub-domain and its boundary conditions

The continuity of the tangential magnetic field between the $i$-th hole and its neighbouring sub-domains (regions I and II) leads to:

$$
\left.\frac{\partial A_{i}}{\partial r}\right|_{r=R_{1}}=\left.\frac{\partial A_{I}}{\partial r}\right|_{r=R_{1}} \text { and }\left.\quad \frac{\partial A_{i}}{\partial r}\right|_{r=R_{2}}=\left.\frac{\partial A_{I I}}{\partial r}\right|_{r=R_{2}}
$$


The general solution of (5) can be found by using the separation of variables method [7] and is given by:

$$
A_{i}(r, \theta)=\sum_{n=1}^{\infty}\left(C_{n}^{i} r^{-\frac{n \pi}{\beta}}+D_{n}^{i} r^{\frac{n \pi}{\beta}}\right) \sin \left(\frac{n \pi}{\beta}\left(\theta-\theta_{i}\right)\right)
$$

where $C_{n}^{i}$ and $D_{n}^{i}$ are the integration constants which are determinated by the interface conditions.

Considering the interface conditions (6) and (7), the general solution of the magnetic vector potential in the $i$-th hole domain can be rewritten as:

$$
A_{i}(r, \theta)=\sum_{n=1}^{\infty}\left(C_{n}^{i} \frac{\beta R_{1}}{n \pi} \frac{P_{n \pi / \beta}\left(r, R_{2}\right)}{E_{n \pi / \beta}\left(R_{1}, R_{2}\right)}-D_{n}^{i} \frac{\beta R_{2}}{n \pi} \frac{P_{n \pi / \beta}\left(r, R_{1}\right)}{E_{n \pi / \beta}\left(R_{2}, R_{1}\right)}\right) \sin \left(\frac{n \pi}{\beta}\left(\theta-\theta_{i}\right)\right)
$$

where $n$ is a positive integer, $P_{n \pi / \beta}$ and $\mathrm{E}_{n \pi / \beta}$ are defined by (3) and (4) respectively. The constants $C_{n}^{i}$ and $D_{n}^{i}$ are obtained using a Fourier series expansion of $\partial A_{I} /\left.\partial r\right|_{R_{1}}$ and $\partial A_{I I} /\left.\partial r\right|_{R_{2}}$ over the hole-opening interval $\left[\theta_{i}, \theta_{i}+\beta\right]$.

$$
\begin{aligned}
& C_{n}^{i}=\left.\frac{2}{\beta} \int_{\theta_{i}}^{\theta_{i}+\beta} \frac{\partial A_{I}}{\partial r}\right|_{R_{1}} \sin \left(\frac{n \pi}{\beta}\left(\theta-\theta_{i}\right)\right) d \theta \\
& D_{n}^{i}=\left.\frac{2}{\beta} \int_{\theta_{i}}^{\theta_{i}+\beta} \frac{\partial A_{I I}}{\partial r}\right|_{R 2} \sin \left(\frac{n \pi}{\beta}\left(\theta-\theta_{i}\right)\right) d \theta
\end{aligned}
$$

The expressions for the coefficients $C_{n}^{i}$ and $D_{n}^{i}$ are given in the appendix.

\subsection{General solution of Laplace's equation in the the rotor shaft region (region I)}

The rotor shaft sub-domain and the associated boundary conditions are shown in Fig. 4. We have to solve Laplace's equation in a circle of radius $R_{l}$ :

$$
\frac{\partial^{2} A_{I}}{\partial r^{2}}+\frac{1}{r} \frac{\partial A_{I}}{\partial r}+\frac{1}{r^{2}} \frac{\partial^{2} A_{I}}{\partial \theta^{2}}=0 \quad \text { for } \quad\left\{\begin{array}{l}
0 \leq r \leq R_{1} \\
0 \leq \theta \leq 2 \pi
\end{array}\right.
$$

The boundary condition at the radius $r=R_{l}$ is difficult to handle because of the existence of the holes as shown in Fig. 1. Considering the continuity of the magnetic vector potential at the interface between the holes and the rotor shaft domains and considering that the magnetic vector potential is equal to zero elsewhere (diamagnetic properties of the superconducting bulks), the boundary condition at $r=R_{l}$ can be written as:

$$
A_{I}\left(R_{1}, \theta\right)=F(\theta)
$$

with

$$
F(\theta)= \begin{cases}A_{i}\left(R_{1}, \theta\right) & \forall \theta \in\left[\theta_{i}, \theta_{i}+\beta\right] \\ 0 & \text { elsewhere }\end{cases}
$$

where $A_{i}(r, \theta)$ is the magnetic vector potential in the $i$-th hole region which is given by (9).

The general solution of (12) is well known [7]. By taking into account the boundary condition (13) and the fact that the magnetic vector potential must be finite at $r=0$, the general solution of (12) can be written as:

$$
A_{I}(r, \theta)=A_{0}^{I}+\sum_{h=1}^{\infty}\left(\frac{r}{R_{1}}\right)^{h}\left(A_{h}^{I} \cos (h \theta)+C_{h}^{I} \sin (h \theta)\right)
$$


where $h$ is a positive integer. The coefficients $A_{0}^{I}, A_{h}^{I}$ and $C_{h}^{I}$ are determined using a Fourier series expansion of $F(\theta)$ over the interval $[0,2 \pi]$

$$
\begin{aligned}
& A_{0}^{I}=\frac{1}{2 \pi} \sum_{i=1}^{Q} \int_{\theta_{i}}^{\theta_{i}+\beta} A_{i}\left(R_{1}, \theta\right) d \theta \\
& A_{h}^{I}=\frac{2}{2 \pi} \sum_{i=1}^{Q} \int_{\theta_{i}}^{\theta_{i}+\beta} A_{i}\left(R_{1}, \theta\right) \cos (h \theta) d \theta \\
& C_{h}^{I}=\frac{2}{2 \pi} \sum_{i=1}^{Q} \int_{\theta_{i}}^{\theta_{i}+\beta} A_{i}\left(R_{1}, \theta\right) \sin (h \theta) d \theta
\end{aligned}
$$

where $Q$ is the number of HTS bulks in the rotor. It is worth noting that the interaction between the holes on the magnetic field distribution is related by the sum operation on $Q$ in (16), (17) and (18). The expressions for the coefficients $A_{0}^{I}, A_{h}^{I}$ and $C_{h}^{I}$ are given in the appendix.

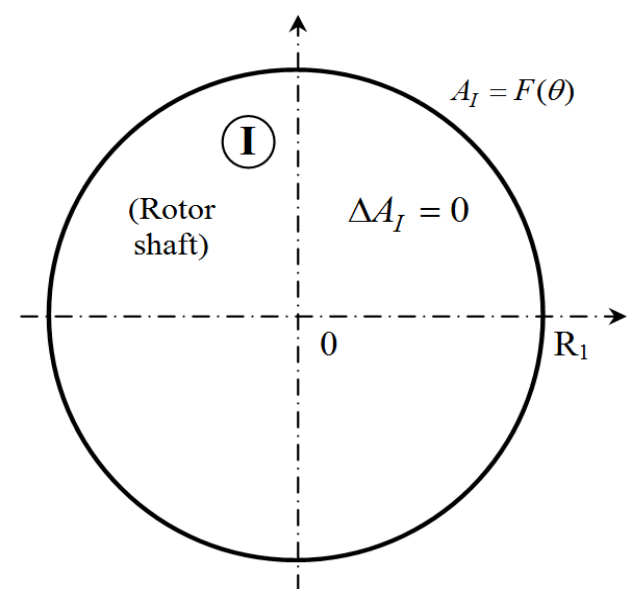

Fig. 4. Rotor shaft sub-domain (region I) with its boundary conditions.

\subsection{General solution of Laplace's equation in the air-gap sub-domain (region II)}

The air-gap sub-domain with its boundary conditions are shown in Fig. 5. We have to solve the laplace equation in an annulus of inner radius $R_{2}$ and outer radius $R_{3}$ :

$$
\frac{\partial^{2} A_{I I}}{\partial r^{2}}+\frac{1}{r} \frac{\partial A_{I I}}{\partial r}+\frac{1}{r^{2}} \frac{\partial^{2} A_{I I}}{\partial \theta^{2}}=0 \quad \text { for } \quad\left\{\begin{array}{l}
R_{2} \leq r \leq R_{3} \\
0 \leq \theta \leq 2 \pi
\end{array}\right.
$$

The same method as the one presented previously is used to determine the boundary condition at $r=R_{2}$. Introducing the function $G(\theta)$, the boundary condition is:

$$
A_{I I}\left(R_{2}, \theta\right)=G(\theta)
$$

with

$$
G(\theta)= \begin{cases}A_{i}\left(R_{2}, \theta\right) & \forall \theta \in\left[\theta_{i}, \theta_{i}+\beta\right] \\ 0 & \text { elsewhere }\end{cases}
$$

where $A_{i}(r, \theta)$ is the magnetic vector potential in the $i$-th hole region which is given by (9).

The boundary condition at $r=R_{3}$ is given by: 


$$
\left.\frac{\partial A_{I I}}{\partial r}\right|_{r=R_{3}}=\mu_{0} j_{s}(\theta)
$$

By taking into account the boundary conditions (20) and (22), the general solution of the magnetic vector potential in the air-gap is given by:

$$
\begin{aligned}
A_{I I}(r, \theta)=A_{0}^{I I} & +\sum_{j=1}^{\infty}\left(A_{j}^{I I} \frac{R_{3}}{j} \frac{E_{j}\left(r, R_{2}\right)}{P_{j}\left(R_{3}, R_{2}\right)}+B_{j}^{I I} \frac{P_{j}\left(r, R_{3}\right)}{P_{j}\left(R_{2}, R_{3}\right)}\right) \cos (j \theta) \\
& +\sum_{j=1}^{\infty}\left(C_{j}^{I I} \frac{R_{3}}{j} \frac{E_{j}\left(r, R_{2}\right)}{P_{j}\left(R_{3}, R_{2}\right)}+D_{j}^{I I} \frac{P_{j}\left(r, R_{3}\right)}{P_{j}\left(R_{2}, R_{3}\right)}\right) \sin (j \theta)
\end{aligned}
$$

where $j$ is a positive integer.

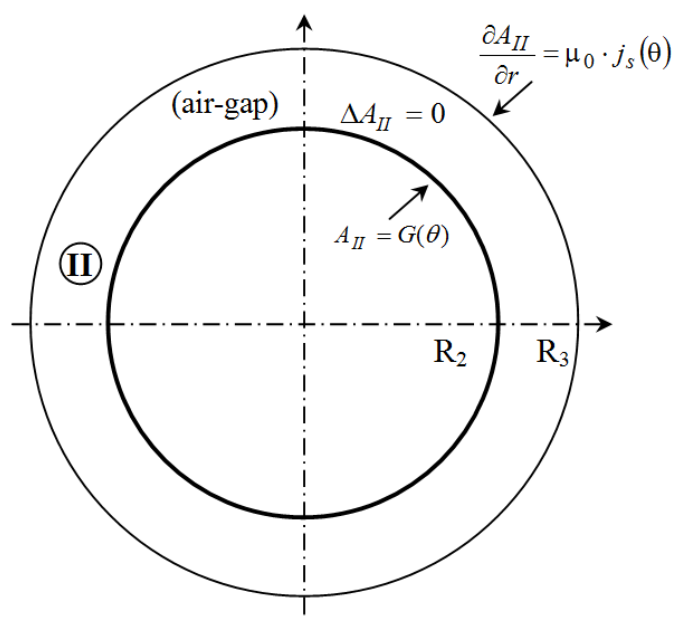

Fig. 5. Air-gap sub-domain (region II) with its boundary conditions.

The integration constants $A_{0}^{I I}, A_{j}^{I I}, B_{j}^{I I}, C_{j}^{I I}$ and $D_{j}^{I I}$ are determined using Fourier series expansions of $\mu_{0} j_{s}(\theta)$ and $G(\theta)$ over the air-gap interval $[0,2 \pi]$ :

$$
\begin{aligned}
& A_{0}^{I I}=\frac{1}{2 \pi} \int_{0}^{2 \pi} G(\theta) d \theta \\
& A_{j}^{I I}=\frac{2}{2 \pi} \int_{0}^{2 \pi} \mu_{0} J_{0} \cos (p \theta) \cos (j \theta) d \theta \\
& B_{j}^{I I}=\frac{2}{2 \pi} \int_{0}^{2 \pi} G(\theta) \cos (j \theta) d \theta \\
& C_{j}^{I I}=\frac{2}{2 \pi} \int_{0}^{2 \pi} \mu_{0} J_{0} \cos (p \theta) \sin (j \theta) d \theta \\
& D_{j}^{I I}=\frac{2}{2 \pi} \int_{0}^{2 \pi} G(\theta) \sin (j \theta) d \theta
\end{aligned}
$$

where the expressions for these coefficients are given in the appendix.

The flux density distribution in the air-gap can be deduced from the magnetic vector potential by 


$$
B_{I I r}=\frac{1}{r} \frac{\partial A_{I I}}{\partial \theta} \quad \text { and } \quad B_{I I \theta}=-\frac{\partial A_{I I}}{\partial r}
$$

The electromagnetic torque is obtained using the Maxwell stress tensor. A circle of radius $R_{e}$ in the airgap sub-domain is taken as the integration path. The electromagnetic torque is then expressed as follows:

$$
\Gamma_{e}=\frac{L R_{e}^{2}}{\mu_{0}} \int_{0}^{2 \pi} B_{I I r}\left(R_{e}, \theta\right) B_{I I \theta}\left(R_{e}, \theta\right) d \theta
$$

where $L$ is the axial length of the superconducting reluctance motor.

\section{Results and comparison with finite element}

In order to validate the proposed analytical model, a comparison with 2D finite element (FE) simulations has been done using FEMM software [13]. The geometrical parameters of the studied HTS reluctance motor are given in Table I. The geometrical parameters given in Table I have been chosen arbitrary. The main objective of this paper is to show the validity of the proposed analytical model.

The analytical solutions in the different regions have been computed with a finite number of harmonic terms $N$ and $K$ as indicated in Table I. In the finite-element simulations, the surfaces of the HTS bulks have been modeled by homogeneous Dirichlet boundary conditions $(A=0)$ as in the analytical study. The mesh in the different regions have been refined until convergent results are obtained.

Table I

Parameters of the model

\begin{tabular}{lll}
\hline Symbol & Quantity & Value \\
\hline$R_{1}$ & Inner radius of bulks & $9 \mathrm{~cm}$ \\
$R_{2}$ & Outer radius of bulks & $9.5 \mathrm{~cm}$ \\
$R_{3}$ & Radius of the current sheet & $10 \mathrm{~cm}$ \\
$\beta$ & HTS bulk opening & $\pi / \mathrm{Q}$ \\
$J_{0}$ & Maximum value of the current sheet & $600 \mathrm{~A} / \mathrm{mm}$ \\
$p$ & Pole-pairs number & 1 \\
$Q$ & Number of bulks & 2 \\
$N$ & Number of harmonics in regions $i$ & 50 \\
$K$ & Number of harmonics in region I and II & 50 \\
\hline
\end{tabular}

\subsection{Flux density distribution}

The magnetic flux lines for two rotor positions $\left(\theta_{0}=0^{\circ}\right.$ and $\left.\theta_{0}=45^{\circ}\right)$ are shown respectively in Fig. 6a-b. The position $\theta_{0}=45^{\circ}$ corresponds to the maximum torque position. The HTS bulks are represented in green color. As expected, the flux lines are repulsed by the superconducting bulks.

The corresponding flux density distributions (radial and tangential components) in the middle of the air-gap (at $r=9.75 \mathrm{~cm}$ ) under no-load condition $\left(\theta_{0}=0^{\circ}\right)$ and load condition $\left(\theta_{0}=45^{\circ}\right)$ are plotted, respectively, in Fig. 7 and Fig. 8. The effect of the HTS bulks on the flux density waveforms is very clear.

One can see the distortion of the flux density distribution at the location of the HTS bulks. The radial flux density is almost null behind the bulks due to the diamagnetic behavior of the superconducting material. One can observe a very good agreement between the analytical and the finite elements predictions for both radial and tangential components. 


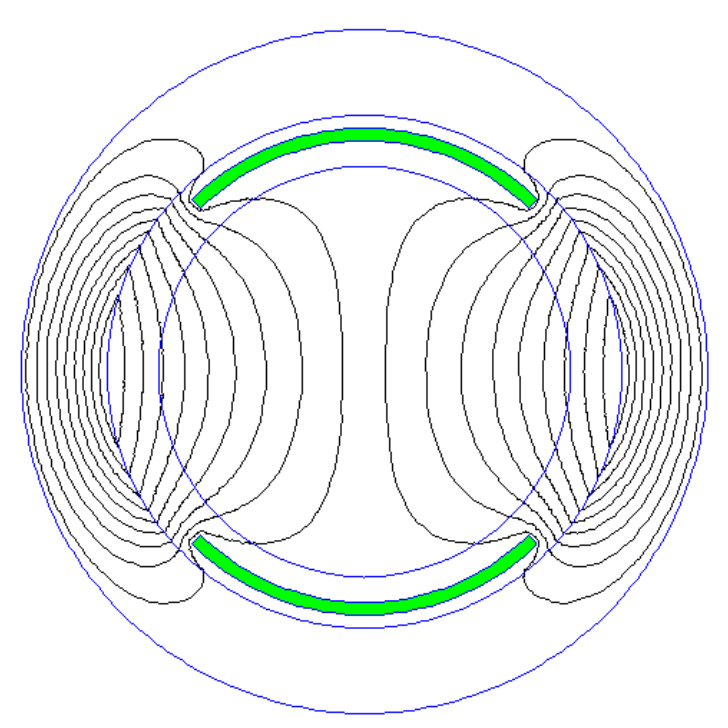

(a)

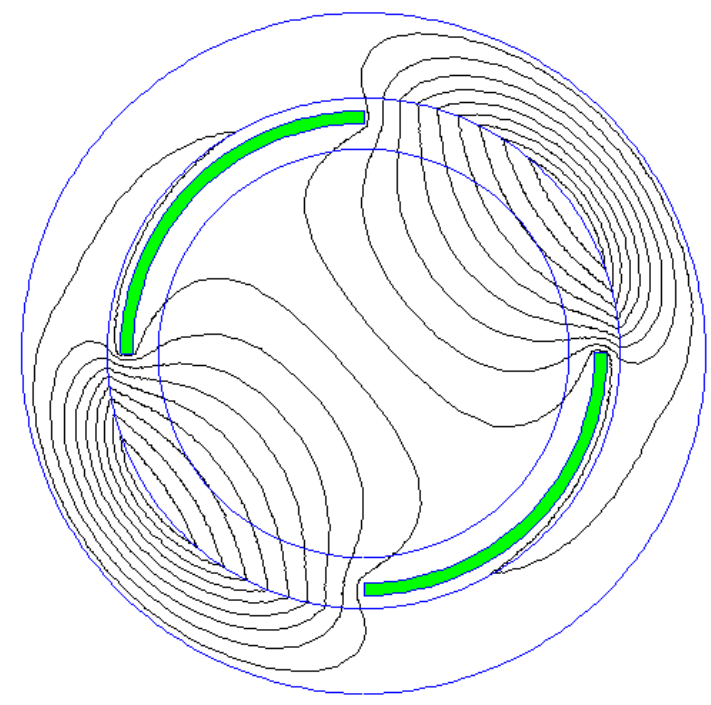

(b)

Fig. 6. Flux lines under (a): no-load condition $\left(\theta_{0}=0^{\circ}\right)$ and (b): load condition $\left(\theta_{0}=45^{\circ}\right)$.

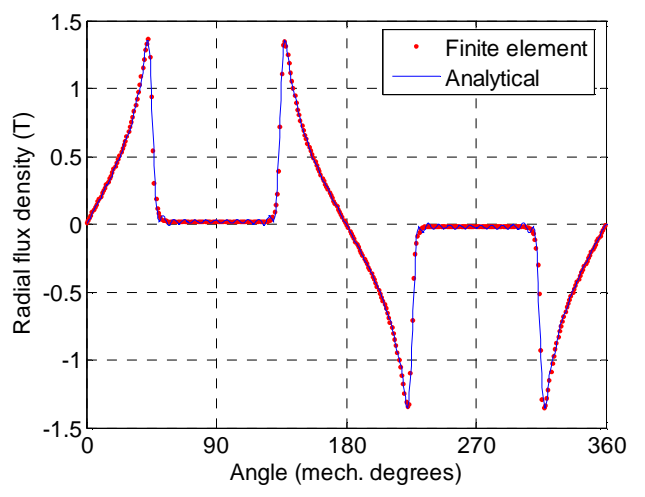

(a)

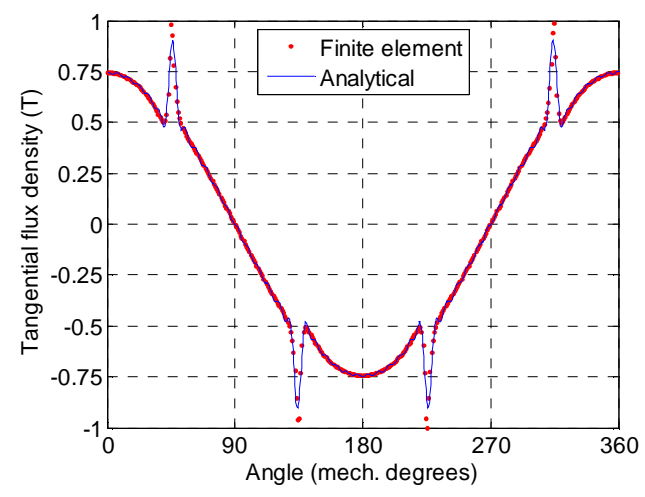

(b)

Fig. 7. Flux density distribution for radial (a) and tangential (b) components in the middle of the airgap domain under no-load condition $\left(\theta_{0}=0^{\circ}\right)$.

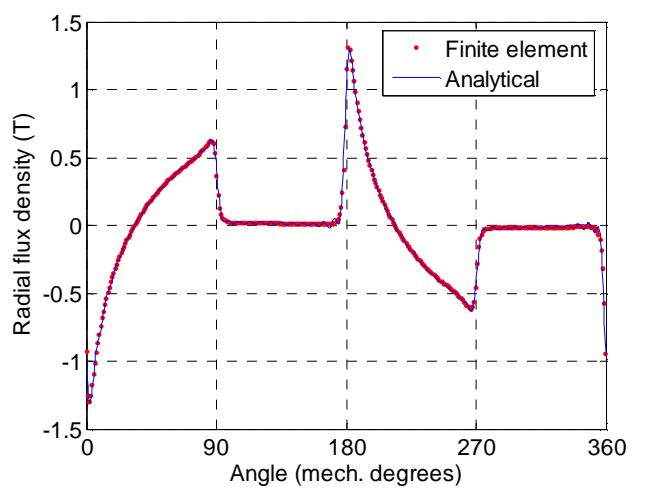

(a)

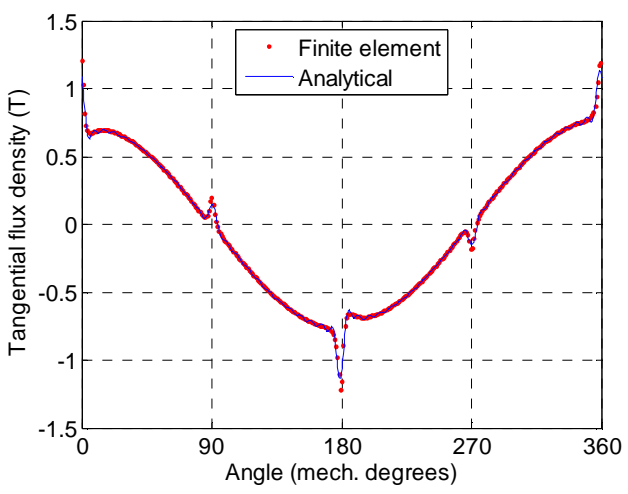

(b)

Fig. 8. Flux density distribution for radial (a) and tangential (b) components in the middle of the airgap domain under load condition $\left(\theta_{0}=45^{\circ}\right)$. 


\subsection{Electromagnetic torque}

The static torque versus mechanical rotor position $\theta_{0}$ is presented in Fig. 9. Compared to the FE simulations, one can see that the analytical calculation well tracks the electromagnetic torque. The torque characteristic shown in Fig. 9 presents the same behavior as conventional 2-pole Synchronous Reluctance Motors [11]. One can observe that a maximum per length torque of around $2700 \mathrm{~N} . \mathrm{m} / \mathrm{m}$ is obtained for a value of $\theta_{0}$ equal to $45^{\circ}$. This corresponds to an active torque density of about $60 \mathrm{kNm} / \mathrm{m}^{3}$ (if we consider an outer radius $R=12 \mathrm{~cm}$ for the stator core), a value which is at least twice that of state-of-the-art conventional SRM [2].

In order to have a good precision in the analytical torque evaluation, the number of harmonic terms used in the computations is equal to $N=50$ (regions $i$ ) and $K=50$ (regions $I$ and $I I$ ) as indicated in Table I. The computation time is about $0.25 \mathrm{~s}$ with the analytical model whereas the finite element simulation takes about $3 \mathrm{~s}$ for a mesh of 50000 elements (for one position of the rotor). The analytical computations being much faster, the presented model can advantageously be used in a preliminary design of HTS reluctance motor.

We have also included in Fig.9 the torque vs. position curve while considering the real $E$ - $J$ power law, viz. $E(J)=E_{c}\left(J / J_{c}\right)^{n}$, of the HTS bulks. The computation is done for $E_{\mathrm{c}}=10^{-4} \mathrm{~V} / \mathrm{m}, \mathrm{J}_{\mathrm{c}}=100 \mathrm{~A} / \mathrm{mm}^{2}$ and $\mathrm{n}=30$, which represent realistic values of YBCO bulks at a critical temperature of $30 \mathrm{~K}$. The problem is solved with 2-D finiteelement software (COMSOL multiphysics). The obtained pull-out torque is about $2280 \mathrm{Nm} / \mathrm{m}$, a value which is $15 \%$ lower when compared to the one obtained with the analytical model that uses the perfect diamagnetism of the HTS bulks. This result shows the ability of the analytical model in predicting the torque of the ScSynRM.

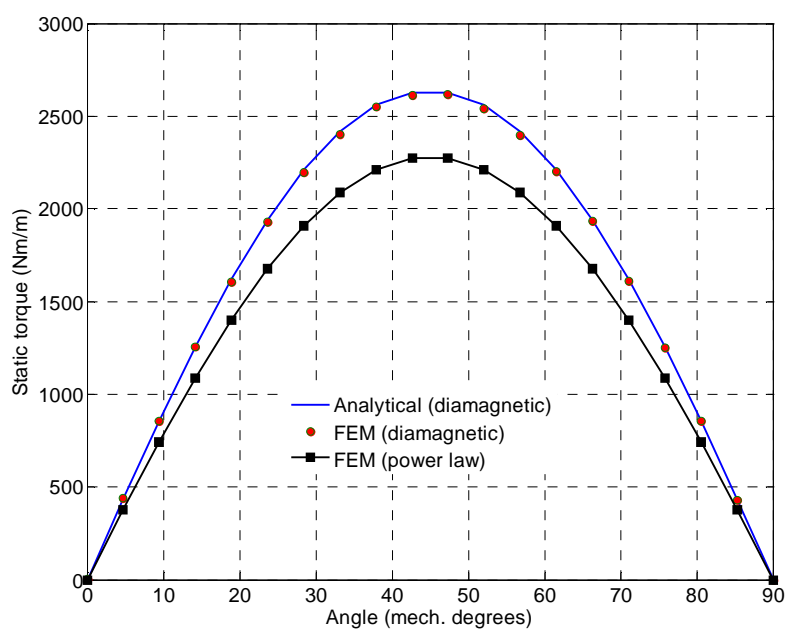

Fig. 9 Static torque versus rotor position for $J_{0}=600 \mathrm{~A} / \mathrm{mm}$ for $p=1$.

\subsection{Effects of pole-pair number and airgap length on the torque value}

The variation of the static torque versus the electrical rotor position $p \theta_{0}$ for different values of the number of pole pairs is shown in Fig. 10. The results have been computed by using (29) and (30). The geometrical parameters are those given in Table I. As it can be observed in Fig. 10, the maximum torque decreases rapidly with the number of pole pairs. The optimum value corresponds to $p=1$ and then $Q=2$ ( $Q$ must be equal to $2 p$ for this reluctance motor).

Fig. 11 shows the variation of the maximum torque as a function of the airgap length for $p=1$. As expected, it can be observed that increasing the airgap length results in lower values of the maximum torque. However, the decreasing is relatively small compared to the large variation of the airgap length. It can be noted that the maximum torque varies approximately linearly with the airgap length. 


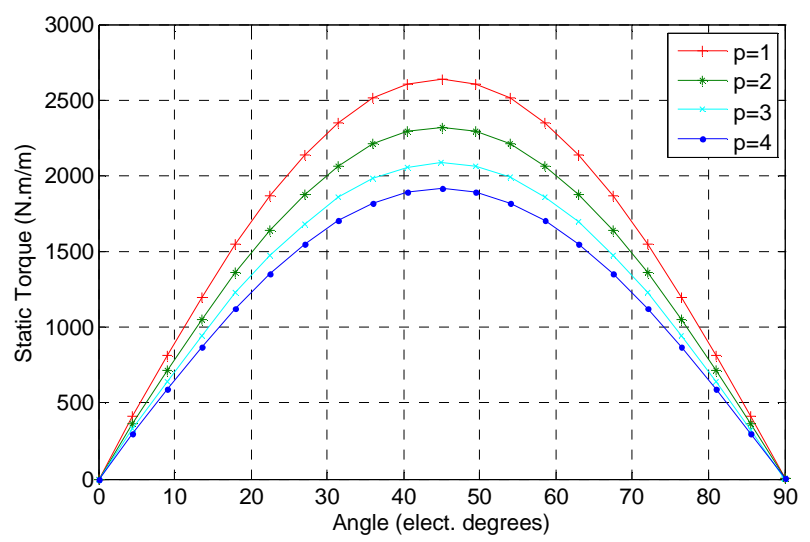

Fig. 10. Effect of the pole-pair number on the torque value.

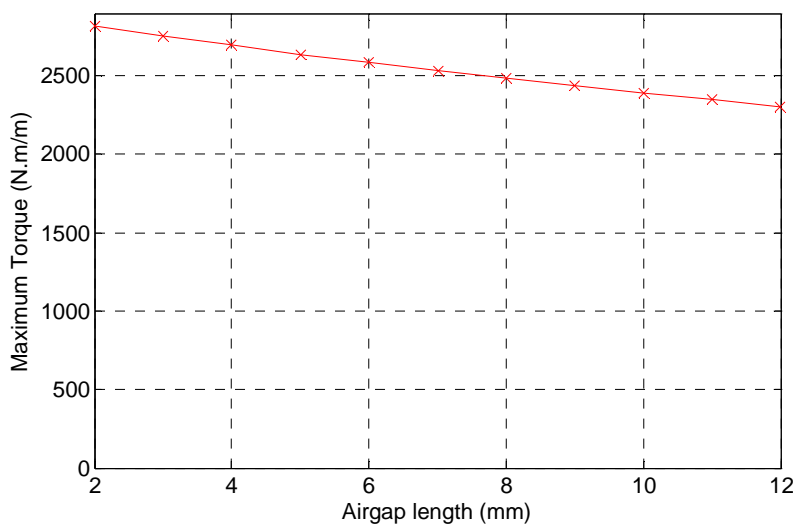

Fig. 11. Effect of the air gap length on the maximum torque for $p=1$.

\section{Conclusion}

In this paper, we have developed an analytical model to calculate the magnetic field distribution and the electromagnetic performances of a superconducting reluctance motor. The proposed motor uses superconducting materials for both the stator and the rotor. The superconducting stator winding can generate a rotating magnetic field of high amplitude without the need of ferromagnetic material in the rotor. The magnetic field generated by the stator interacts with the HTS bulks rotor. The electromagnetic torque is obtained by the magnetic flux lines deviation due to the flux shielding capability of the HTS bulks rotor (reluctance effect).

The proposed model is based on boundary value problems with Fourier analysis. To simplify the analysis, we have considered the HTS bulks as a perfect diamagnetic material, therefore homogeneous Dirichlet boundary condition $A=0$ has been imposed on the surface of the HTS bulks. To show the effectiveness of the proposed model, we have compared the results with those obtained by finite element simulations. We have shown that the proposed analytical model is able to predict the performances of the proposed HTS motor. Moreover, the analytical model presents less computational time than numerical ones. Hence, it can be used as a preliminary tool to investigate the influence of the design parameters on the motor performances or for design optimisation of the studied machine.

\section{Appendix}

For the determination of the integration coefficients, we have to calculate integrals of the form:

$$
\begin{aligned}
& f_{u}(n, i)=\int_{\theta_{i}}^{\theta_{i}+\beta} \cos (u \theta) \sin \left(\frac{n \cdot \pi}{\beta}\left(\theta-\theta_{i}\right)\right) d \theta \\
& g_{u}(n, i)=\int_{\theta_{i}}^{\theta_{i}+\beta} \sin (u \theta) \sin \left(\frac{n \pi}{\beta}\left(\theta-\theta_{i}\right)\right) d \theta
\end{aligned}
$$




$$
N_{n}=\int_{\theta_{i}}^{\theta_{i}+\beta} \sin \left(\frac{n \cdot \pi}{\beta}\left(\theta-\theta_{i}\right)\right) d \theta
$$

The development of these integrals, which are used in the expressions of the integration coefficients, are given by:

$$
\begin{aligned}
& \text { - for } n \pi \neq u \beta \\
& \qquad \begin{aligned}
f_{u}(n, i) & =\frac{n \pi \beta}{(u \beta)^{2}-(n \pi)^{2}}\left((-1)^{n} \cos \left(\left(\theta_{i}+\beta\right) u\right)-\cos \left(\theta_{i} u\right)\right) \\
g_{u}(n, i) & =\frac{n \pi \beta}{(u \beta)^{2}-(n \pi)^{2}}\left((-1)^{n} \sin \left(\left(\theta_{i}+\beta\right) u\right)-\sin \left(\theta_{i} u\right)\right)
\end{aligned}
\end{aligned}
$$

- for $n \pi=u \beta$

$$
\begin{aligned}
& f_{u}(n, i)=-\frac{\beta}{2}\left(\sin \left(u \theta_{i}\right)+\frac{1}{2 n \pi}\left(\cos \left(\left(\theta_{i}+2 \beta\right) u\right)-\cos \left(\theta_{i} u\right)\right)\right) \\
& g_{u}(n, i)=\frac{\beta}{2}\left(\cos \left(u \theta_{i}\right)-\frac{1}{2 n \pi}\left(\sin \left(\left(\theta_{i}+2 \beta\right) u\right)-\sin \left(\theta_{i} u\right)\right)\right) \\
& N_{n}=\beta \frac{1-(-1)^{n}}{n \pi}
\end{aligned}
$$

- Expressions of the coefficients $A_{0}^{I}, A_{h}^{I}$ and $C_{h}^{I}$ for the rotor shaft sub-domain (region $I$ ).

The development of (16), (17) and (18) gives

$$
\begin{gathered}
A_{0}^{I}=\frac{1}{2 . \pi} \sum_{i=1}^{Q} \sum_{n=1}^{\infty} C_{n}^{i} \frac{\beta R_{1}}{n . \pi} \frac{P_{n \pi / \beta}\left(R_{1}, R_{2}\right)}{E_{n \pi / \beta}\left(R_{1}, R_{2}\right)} N_{n}+\frac{1}{2 . \pi} \sum_{i=1}^{Q} \sum_{n=1}^{\infty} D_{n}^{i} \frac{\beta R_{2}}{n . \pi} \frac{2}{E_{n \pi / \beta}\left(R_{2}, R_{1}\right)} N_{n} \\
A_{h}^{I}=\frac{1}{\pi} \sum_{i=1}^{Q} \sum_{n=1}^{\infty} C_{n}^{i} \frac{\beta R_{1}}{n . \pi} \frac{P_{n \pi / \beta}\left(R_{1}, R_{2}\right)}{E_{n \pi / \beta}\left(R_{1}, R_{2}\right)} f_{u}(n, i)+\frac{1}{\pi} \sum_{i=1}^{Q} \sum_{n=1}^{\infty} D_{n}^{i} \frac{\beta R_{2}}{n . \pi} \frac{2}{E_{n \pi / \beta}\left(R_{2}, R_{1}\right)} f_{u}(n, i) \\
C_{h}^{I}=\frac{1}{\pi} \sum_{i=1}^{Q} \sum_{n=1}^{\infty} C_{n}^{i} \frac{\beta R_{1}}{n . \pi} \frac{P_{n \pi / \beta}\left(R_{1}, R_{2}\right)}{E_{n \pi / \beta}\left(R_{1}, R_{2}\right)} g_{u}(n, i)+\frac{1}{\pi} \sum_{i=1}^{Q} \sum_{n=1}^{\infty} D_{n}^{i} \frac{\beta R_{2}}{n . \pi} \frac{2}{E_{n \pi / \beta}\left(R_{2}, R_{1}\right)} g_{u}(n, i)
\end{gathered}
$$

- Expressions $C_{n}^{i}$ and $D_{n}^{i}$ for the $i$-th hole sub-domain (regions $i$ ).

The development of (10) and (11) gives

$$
\begin{aligned}
& C_{n}^{i}=\frac{2}{\beta} \sum_{h=1}^{\infty}\left(A_{h}^{I} \frac{h}{R_{1}} f_{u}(n, i)+C_{h}^{I} \frac{h}{R_{1}} g_{u}(n, i)\right) \\
& D_{n}^{i}=-\frac{B_{0}^{I I} N_{n}}{R_{2}} \frac{2}{\beta}+\frac{2}{\beta} \sum_{j=1}^{\infty}\left(A_{j}^{I I} \frac{R_{3}}{R_{2}} \frac{2}{P_{j}\left(R_{3}, R_{2}\right)}+B_{j}^{I I} \frac{j}{R_{2}} \frac{E_{j}\left(R_{2}, R_{3}\right)}{P_{j}\left(R_{2}, R_{3}\right)}\right) f_{u}(n, i) \\
& +\frac{2}{\beta} \sum_{j=1}^{\infty}\left(C_{j}^{I I} \frac{R_{3}}{R_{2}} \frac{2}{P_{j}\left(R_{3}, R_{2}\right)}+D_{j}^{I I} \frac{j}{R_{2}} \frac{E_{j}\left(R_{2}, R_{3}\right)}{P_{j}\left(R_{2}, R_{3}\right)}\right) g_{u}(n, i)
\end{aligned}
$$

- Expressions $A_{0}^{I I}, A_{j}^{I I}, B_{j}^{I I}, C_{j}^{I I}$ and $D_{j}^{I I}$ for the air-gap sub-domain (region $\left.I I\right)$. 
The development of (24) to (28) gives:

$$
\begin{aligned}
& A_{0}^{I I}=\frac{1}{2 . \pi} \sum_{i=1}^{Q} \sum_{n=1}^{\infty} C_{n}^{i} \frac{\beta \cdot R_{1}}{n . \pi} \frac{2}{E_{n \pi / \beta}\left(R_{1}, R_{2}\right)} N_{n}+\frac{1}{2 . \pi} \sum_{i=1}^{Q} \sum_{n=1}^{\infty} D_{n}^{i} \frac{\beta \cdot R_{2}}{n . \pi} \frac{P_{n \pi / \beta}\left(R_{2}, R_{1}\right)}{E_{n \pi / \beta}\left(R_{2}, R_{1}\right)} N_{n} \\
& A_{j=p}^{I I}=\mu_{0} J_{0} \\
& B_{j}^{I I}=\frac{1}{\pi} \sum_{i=1}^{Q} \sum_{n=1}^{\infty} C_{n}^{i} \frac{\beta R_{1}}{n . \pi} \frac{2}{E_{n \pi / \beta}\left(R_{1}, R_{2}\right)} f_{u}(n, i)+\frac{1}{\pi} \sum_{i=1}^{Q} \sum_{n=1}^{\infty} D_{n}^{i} \frac{\beta R_{2}}{n . \pi} \frac{P_{n \pi / \beta}\left(R_{2}, R_{1}\right)}{E_{n \pi / \beta}\left(R_{2}, R_{1}\right)} f_{u}(n, i) \\
& C_{j}^{I I}=0 \\
& D_{j}^{I I}=\frac{1}{\pi} \sum_{i=1}^{Q} \sum_{n=1}^{\infty} C_{n}^{i} \frac{\beta R_{1}}{n . \pi} \frac{2}{E_{n \pi / \beta}\left(R_{1}, R_{2}\right)} g_{u}(n, i)+\frac{1}{\pi} \sum_{i=1}^{Q} \sum_{n=1}^{\infty} D_{n}^{i} \frac{\beta R_{2}}{n . \pi} \frac{P_{n \pi / \beta}\left(R_{2}, R_{1}\right)}{E_{n \pi / \beta}\left(R_{2}, R_{1}\right)} g_{u}(n, i)
\end{aligned}
$$

From (A.9) to (A.18), we have found the expressions of the ten coeffients (one of the coefficient is null i.e. A.17). To obtain the solution of the magnetic field in the different sub-domains, a system of linear equations has to be solved. The total number of equation depends on the number of harmonic terms used in each sub-domain. By rewriting the above equations in matrix and vectors format, a numerical solution can be found by using mathematical software (Matlab).

\section{References}

[1] A. Bellara, Y. Amara, G. Barakat, B. Dakyo, Two-dimensional exact analytical solution of armature reaction field in slotted surface mounted PM radial flux synchronous machines, IEEE Trans. Magn. 45 (10) (2009) 4534-4538.

[2] I. Boldea Reluctance synchronous machines and drives, Oxford University Press, London, 1996.

[3] E. H. Brandt, Superconductor of finite thickness in a perpendicular magnetic field: strips and slabs, Physical Review B 54 (1996) 42464264.

[4] A. Chebak, P. Viarouge, J. Cros, Optimal design of a high-speed slotless permanent magnet synchronous generator with soft magnetic composite stator yoke and rectifier load, Math. Comput. Simul. 81 (2) (2010) 239-251.

[5] S. D. Chu, S. Torii, Torque-speed characteristics of superconducting synchronous reluctance motors with DyBCO bulk in the rotor, IEEE Trans. Appl. Supercond. 15 (2) (2005) 2178-2181.

[6] F. Dubas, C. Espanet, Analytical solution of the magnetic field in permanent-magnet motors taking into account slotting effect: no-load vector potential and flux density calculation, IEEE Trans. Magn. 45 (5) (2009) 2097-21092.

[7] S.J. Farlow, Partial differential equations for scientists and engineers, Dover, Mineola, New York, 1993.

[8] B. L. J. Gysen, K. J. Meessen, J. J. H. Paulides, E. A. Lomonova, General formulation of the electromagnetic field distribution in machines and devices using Fourier analysis, IEEE Trans. Magn. 46 (1) (2010) 4639-4652.

[9] L. K. Kovalev, K. V. Ilushin, S. M-A. Koneev, K. L. Kovalev, V. T. Penkin, V. N. Poltavets, W. Gawalek, T. Habisreuther, B. Oswald, K-J. Best, Hysteresis and reluctance electric machines with bulk HTS rotor elements, IEEE Trans. Appl. Supercond. 9 (2) (1999) 12611264.

[10] Z. J. Liu, J. T. Li, Accurate prediction of magnetic field and magnetic forces in permanent magnet motor using an analytical solution, IEEE Trans. Energy. Convers. 23 (3) (2008) 717-726.

[11] T. Lubin, S. Mezani, A. Rezzoug, Exact analytical method for magnetic field computation in the air-gap of cylindrical electrical machines considering slotting effects, IEEE Trans. Magn. 46 (4) (2010) 1092-1099.

[12] G. Malé, S. Mezani, T. Lubin, J. Lévêque, A fast analytical method to compute the radial flux density distribution in the airgap of a superconducting inductor, IEEE Trans. Appl. Supercond. 21 (3) (2011) 1114-1118.

[13] D.C. Meeker, Finite element method magnetics, version 4.2, (1 April 2009 build), http://www.femm.info

[14] B. Oswald, K-J. Best, M. Setzer, M. Söll, W. Gawalek, A. Gutt, L. Kovalev, G. Krabbes, L. Fisher, H. C. Freyhardt, Reluctance motors with bulk HTS material, Supercond. Sci. Technol. 18 (2005) S24-S29.

[15] R. Schiferl, A. Flory, W.C. Livoti, S.D. Umans, High-temperature superconducting synchronous motors: economic issues for industrial applications, IEEE Trans. Appl. Supercond. 44 (5) (2008) 1376-1384.

[16] H. Tiegna, Y. Amara, G. Barakat, Analytical modeling of permanent magnet electric machines for analysis and design purpose, Math. Comput. Simul., this issue.

[17] Z.Q. Zhu, L.J. Wu, Z.P. Xia, An accurate subdomain model for magnetic field computation in slotted surface-mounted permanent magnet machines, IEEE Trans. Magn. 46 (4) (2010) $1100-1115$.

[18] Sumitomo Electric, Superconductivity. Internet: http://globalsei.com/super/hts e/type h.html,

[19] Can Superconductors. Internet: http://www.can-superconductors.com/ 